\title{
High glucose induces apoptosis of HUVECs in a mitochondria-dependent manner by suppressing hexokinase 2 expression
}

\author{
JIA ZHANG $^{1,2 *}$, YANYAN GUO ${ }^{3 *}$, WANYU GE $^{4}$, XIA ZHOU $^{5}$ and MINGLIN PAN ${ }^{5}$ \\ ${ }^{1}$ Sir Run Run Hospital, Nanjing Medical University, Jiangsu, Nanjing 211100; ${ }^{2}$ Department of Pharmacology, School of \\ Basic Medical Science, Nanjing Medical University, Jiangsu, Nanjing 211166; ${ }^{3}$ Department of General Medicine, \\ Baota Bridge Community Health Service Center, Jiangsu, Nanjing 210011; ${ }^{4}$ Department of Pathology, \\ Anhui Provincial Hospital, Hefei, Anhui 230000; ${ }^{5}$ Department of Endocrinology, The Second \\ Affiliated Hospital of Nanjing Medical University, Jiangsu, Nanjing 210011, P.R. China
}

Received April 16, 2018; Accepted April 14, 2019

DOI: $10.3892 /$ etm.2019.7609

\begin{abstract}
Hyperglycemia in patients with diabetes induces vascular endothelial cell apoptosis and subsequent vasculopathy. The aim of the current study was to investigate the pathological mechanism of hyperglycemia-induced endothelial cell apoptosis and vasculopathy using human umbilical vein endothelial cells. As high glucose-induced apoptosis is caused by elevated mitochondrial permeability-mediated release of mitochondrial cytochrome $\mathrm{c}$, the current study examined voltage-dependent anion channel (VDAC1), the controller of mitochondrial permeability, and its regulators, hexokinase2 (HK2), Bcl-2 and Bax. The current study demonstrated that HK2 may be involved in high glucose-induced cell apoptosis, as HK2 overexpression partially reversed high glucose-induced downregulation of mitochondrial/cellular HK2 and Bcl-2 as well as upregulation of mitochondrial Bax. These results suggest that HK 2 overexpression partially reversed the reduced binding of $\mathrm{HK} 2$ and $\mathrm{Bcl}-2$ and the enhanced binding of Bax to VDAC1, which reduced the high mitochondrial permeability observed under high-glucose conditions. Furthermore, high glucose reduced HK2 transcription via down-regulation of the HK2 transcriptional factor,
\end{abstract}

Correspondence to: Professor Minglin Pan, Department of Endocrinology, The Second Affiliated Hospital of Nanjing Medical University, 121 Jiangjiayuan Road, Jiangsu, Nanjing 210011, P.R. China E-mail: panminglin2018@163.com

*Contributed equally

Abbreviations: HK2, hexokinase 2; VDAC1, voltage-dependent anion channel 1; CyA, cyclosporine A; CREB, cyclic AMP response

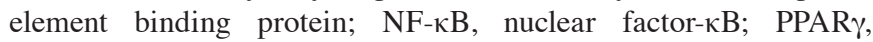
peroxisome proliferator-activated receptor $\gamma$

Key words: hyperglycemia, hexokinase 2, voltage-dependent anion channel 1, Bcl-2, Bcl-2 associated X, mitochondria peroxisome proliferator activated receptor $\gamma(\operatorname{PPAR} \gamma)$. Taken together, these results suggest that PPAR $\gamma / \mathrm{HK} 2$ may be novel targets for the prevention of diabetic vasculopathy.

\section{Introduction}

Diabetes mellitus is a chronic endocrine disorder, characterized by hyperglycemia and other metabolic disorders of fat, protein and electrolytes due to absolute or relative insulin deficiency (1). Epidemiological data suggest that diabetes-induced cardiovascular complications are a leading cause of diabetes-related mortality and disability (2). Diabetes-induced cardiovascular complications include macrovascular and microvascular diseases (3), of which vascular endothelial cell apoptosis is considered to be an initial event and pathological basis (4). However, the underlying mechanism of diabetes-induced endothelial cell apoptosis remains unknown.

Mitochondria, membrane-bound organelles located in the cytoplasm of almost all eukaryotic cells, are commonly referred to as cellular power plants critical to the production of energy in the form of ATP (5). In addition, mitochondria serve a central role in cell apoptosis (6). Damage to the mitochondria leads to increased mitochondrial membrane permeability and subsequent release of cytochrome c (7), an essential component of the electron transport chain (8). Upon entering the cytoplasm, cytochrome c interacts with apoptotic protease activating factor-1 that triggers the activation of caspase cascades (9). Previous studies have confirmed that hyperglycemia induces mitochondrial pathway-dependent apoptosis in vascular endothelial cells (10), however the underlying mechanisms remain unknown.

Increasing evidence suggests that voltage-dependent anion channel (VDAC), an abundant protein located in the outer mitochondrial membrane (11), serves a role in the regulation of mitochondrial membrane permeability and is essential for the release of cytochrome c during cell apoptosis (12). The VDAC protein family consists of 3 isoforms: VDAC1, VDAC2 and VDAC3, and vascular endothelial cells mainly express VDAC1 (13). VDAC provides the pathway for the movement 
of ATP and other small molecules out of the mitochondria (14). Mitochondrial membrane permeability is regulated by VDAC1 together with other factors including hexokinase 2 (HK2), Bcl-2 and Bax (15). HK2 and Bcl-2 decreases, while Bax increases mitochondrial permeability (16-18). Once cells are exposed to apoptogenic factors, such as oxidative stress, this leads to the VDAC1-mediated increase in mitochondrial permeability, with the release of cytochrome $\mathrm{c}$ and subsequent cell apoptosis (19).

The present study investigated the pro-apoptotic effect of high glucose on human umbilical vein endothelial cells (HUVECs), a commonly used cell line in diabetic vascular research (20) and the potential underlying mechanisms. The results of the current study indicated that high glucose may induce HUVEC apoptosis via downregulation of HK2 and $\mathrm{Bcl}-2$ proteins, leading to decreased interactions with VDAC1. Furthermore, increased interactions between Bax and VDAC1 lead to the subsequent release of cytochrome c. Taken together, the results of the current study may provide new approaches and potential targets for the prevention of diabetes-induced cardiovascular complications.

\section{Materials and methods}

Cell culture. HUVECs were purchased from the American Type Culture Collection. HUVECs were cultured in Dulbecco's modified Eagle's medium (DMEM; Thermo Fisher Scientific, Inc.) containing $5.5 \mathrm{mM}$ glucose with $10 \%$ fetal bovine serum (FBS; Thermo Fisher Scientific, Inc.), $100 \mathrm{U} / \mathrm{ml}$ penicillin and $100 \mathrm{mg} / \mathrm{ml}$ streptomycin (Thermo Fisher Scientific, Inc.) at $37^{\circ} \mathrm{C}$ in a $5 \% \mathrm{CO}_{2}$-humidified incubator. MG132 was utilized to prevent the potential proteasome-induced degradation of protein. HUVEC cells were pretreated with $50 \mu \mathrm{M}$ MG132 (Selleck Chemicals) for $8 \mathrm{~h}$ at $37^{\circ} \mathrm{C}$ in a $5 \% \mathrm{CO}_{2}$-humidified incubator before culture in DMEM containing 5.5, 16.5 or $33 \mathrm{mM}$ glucose for a further $72 \mathrm{~h}$ at $37^{\circ} \mathrm{C}$. To examine the effect of cyclosporine A (CyA), HUVECs were treated with $10 \mathrm{mM}$ CyA (EMD Millipore) for $12 \mathrm{~h}$ at $37^{\circ} \mathrm{C}$ in a $5 \%$ $\mathrm{CO}_{2}$-humidified incubator. Following the 12-h treatment, HUVECs were cultured in DMEM containing 5.5 or $33 \mathrm{mM}$ glucose for a further $72 \mathrm{~h}$.

Cell infection. HUVEC cells were seeded into 6-well plates at a density of $4 \times 10^{5}$ cells/well overnight at $37^{\circ} \mathrm{C}$. Virus particles $\left(2 \times 10^{7}\right) /$ well [HK2 adenovirus or the control virus; OBiO Technology (Shanghai) Corp., Ltd.] were subsequently added and incubated for $2 \mathrm{~h}$ at $37^{\circ} \mathrm{C}$. Cells were cultured in fresh DMEM containing 5.5 or $33 \mathrm{mM}$ glucose for a further $72 \mathrm{~h}$ at $37^{\circ} \mathrm{C}$ in a $5 \% \mathrm{CO}_{2}$-humidified incubator.

Vectors. pGL3-HK2, pGL3-Bcl-2, pRL-TK-Renilla plasmid and $\mathrm{pCF}$ CREB, pCMV4 and pSV-SPORT expression vectors were gifts provided by Professor Dongping Wei (The First Affiliated Hospital of Nanjing, Nanjing, China). The mammalian expression vector pcDNA3.1 was purchased from Thermo Fisher Scientific (San Jose, CA, USA). The HK2 adenovirus and the control virus were purchased from Obio Technology Co., Ltd. (Shanghai, China). The pSV Sport PPAR $\gamma$ (Addgene plasmid no. 8886) and pCMV4 p65 (Addgene plasmid no. 21966) plasmids were gifted by Dr Bruce Spiegelman
(Dana-Farber Cancer Institute, Harvard Medical School) and Dr Warner Greene (Howard Hughes Medical Institute, Department of Medicine, Duke University Medical Center), respectively.

Cell transfection. HUVEC cells were seeded into 6-well plates at a density of $4 \times 10^{5}$ cells/well overnight at $37^{\circ} \mathrm{C}$. Cells were subsequently transfected with pCF CREB (pcDNA3.1), pCMV4-p65 (pCMV4),pSV SPORT PPAR $\gamma$ (pSV SPORT) and flag-tagged PPAR $\gamma$ plasmids (1 $\mu \mathrm{g} /$ well) using Lipofectamine ${ }^{\circledR}$ 2000 (Thermo Fisher Scientific, Inc.). A ratio of $1 \mu \mathrm{g}$ plasmid to $2 \mu 1$ liposome was then diluted in serum-free DMEM. After $4 \mathrm{~h}$, cells were cultured in 10\% FBS with DMEM for a further $48 \mathrm{~h}$ at $37^{\circ} \mathrm{C}$ in a $5 \% \mathrm{CO}_{2}$-humidified incubator prior to subsequent experimentation.

Cell viability assay. Cell viability was examined by MTT assay. HUVEC cells were seeded into 96-well plates at a density of $2 \times 10^{4}$ cells/well and cultured for $24 \mathrm{~h}$. Subsequently, cells were cultured in fresh DMEM containing 5.5, 16.5 or $33 \mathrm{mM}$ glucose for a further $72 \mathrm{~h}$ or cells were infected with the HK2 adenovirus or the control virus following culture in fresh DMEM containing $5.5,16.5$ or $33 \mathrm{mM}$ glucose for a further $72 \mathrm{~h}$. Following incubation, MTT solution (Beyotime Institute of Biotechnology) was added to each well at a final concentration of $5 \mathrm{mg} / \mathrm{ml}$ and cells were incubated for $1 \mathrm{~h}$ at $37^{\circ} \mathrm{C}$. The formazan precipitate was dissolved in $200 \mu 1$ DMSO (Thermo Fisher Scientific, Inc.) and the absorbance was measured at $570 \mathrm{~nm}$ using a microplate reader (SpectraFluor; Tecan Group, Ltd.). Cell survival was expressed as a percentage of the absorbance in the control wells.

Cell apoptosis analysis. Cell apoptosis was examined by Hoechst 33258 staining. Cells were washed three times with PBS and fixed in $4 \%$ paraformaldehyde for $\sim 12 \mathrm{~h}$ at $4^{\circ} \mathrm{C}$. Cells were then washed a further three times with PBS and incubated with $10 \mu \mathrm{g} / \mathrm{ml}$ Hoechst 33258 (Beyotime Institute of Biotechnology) was added to each sample for $5 \mathrm{~min}$ at room temperature. Cells were washed with PBS and the coverslips were mounted with glycerol and images were captured using a fluorescence microscope (magnification, $x 200$ ) with an excitation wavelength at $346 \mathrm{~nm}$ and an emission wavelength at $460 \mathrm{~nm}$.

Isolation of mitochondria. HUVEC cells were resuspended in mitochondria isolation reagents (Cell mitochondria separation kit; cat. no. c3601; Beyotime Institute of Biotechnology) and incubated on ice for $15 \mathrm{~min}$. Following homogenization, the cell lysate was centrifuged at $600 \mathrm{xg}$ for $10 \mathrm{~min}$ at $4{ }^{\circ} \mathrm{C}$, and the supernatant was collected and further centrifuged at $11,000 \mathrm{x} \mathrm{g}$ for $10 \mathrm{~min}$ at $4^{\circ} \mathrm{C}$. The remaining mitochondrial pellet was collected and used for subsequent experimentation.

Flow cytometric analysis of mitochondrial membrane potential using JC-1 staining. Following trypsinization, cells were washed twice with PBS, resuspended in $1 \mathrm{ml} \mathrm{JC}-1$ staining solution (1X; Beyotime Institute of Biotechnology) and incubated at $37^{\circ} \mathrm{C}$ for $20 \mathrm{~min}$. Following incubation, cells were centrifuged at $600 \mathrm{xg}$ for $10 \mathrm{~min}$ at $4^{\circ} \mathrm{C}$ and resuspended in $500 \mu 1$ staining buffer (cat. no. c2005; Beyotime Institute of 
Biotechnology). Mitochondrial membrane potential was subsequently examined via flow cytometric analysis with FlowJo VX10 software (FlowJo LLC), where fluorescent emissions were detected at $530 \mathrm{~nm}$ (JC-1 FL1; red) and $575 \mathrm{~nm}$ (JC-1 FL2; green). The FL2/FL1 ratio represents mitochondrial membrane potential.

Immunoprecipitation and western blot analysis. HUVEC cells or mitochondria were lysed using radioimmunoprecipitation assay buffer (20 mM Tris, $150 \mathrm{mM} \mathrm{NaCl}, 1 \%$ Triton X-100 and $1 \mathrm{mM}$ PMSF, pH 7.5) and incubated for $30 \mathrm{~min}$ on ice. Samples were then centrifuged for $10 \mathrm{~min}$ at $13,800 \mathrm{x} \mathrm{g}$ at $4^{\circ} \mathrm{C}$. Lysates were resuspended in $3 \mathrm{X}$ loading buffer (2\% SDS, $6 \% \beta$-mercaptoethanol, $50 \mathrm{mM}$ Tris- $\mathrm{HCl}, 10 \%$ glycerol and $0.05 \%$ bromophenol blue, $\mathrm{pH}$ 6.8). Cell lysates were incubated with primary antibody (as stated below) for $2 \mathrm{~h}$ at $4{ }^{\circ} \mathrm{C}$ followed by incubation with protein A-sepharose beads (Sea Biotech, Shanghai, China) for $1 \mathrm{~h}$ at $4^{\circ} \mathrm{C}$. Protein A-sepharose beads were washed three times with lysis buffer and centrifuged at $4^{\circ} \mathrm{C}$ for $1 \mathrm{~min}$ at $6,200 \mathrm{xg}$. The supernatant was discarded and $2 \mathrm{X}$ loading buffer ( $2 \%$ SDS, $6 \% \beta$-mercaptoethanol, $50 \mathrm{mM}$ Tris- $\mathrm{HCl}, 10 \%$ glycerol and $0.05 \%$ bromophenol blue; $\mathrm{pH} 6.8$ ) was added to the protein A-sepharose beads. Samples were then boiled at $95^{\circ} \mathrm{C}$ for $5 \mathrm{~min}$. Protein quantity was determined using a BCA protein assay kit (Thermo Fisher Scientific, Inc.). Protein $(25 \mu \mathrm{g})$ was separated by $10 \%$ SDS-PAGE and transferred to PDVF membranes (EMD Millipore). Membranes were then blocked with 5\% skimmed milk in TBST for $1 \mathrm{~h}$ at room temperature and incubated with the following primary antibodies: HK2 (1:1,000; cat. no. 2867), $\beta$-actin $(1: 1,000$; cat. no. 3700), Bcl-2 (1:1,000; cat. no. 2872), Bax (1:1,000; cat. no. 5023), cytochrome c (1:1,000; cat. no. 4272), cleaved caspase-3 (1:1,000; cat. no. 9661), PPAR $\gamma$ (1:1,000; cat. no. 2435), Flag (1:1,000; cat. no. 2368), phosphorylated-CREB (1:1,000; cat. no. 9198), CREB (1:1,000; cat. no. 9197), phosphorylated-NF- $\mathrm{B}(1: 1,000$; cat. no. 3033$), \mathrm{NF}-\kappa \mathrm{B}$ (1:1,000; cat. no. 8242; all Cell Signaling Technology, Inc.), COX4 (1:1,000; cat. no. 202554; Abcam) and VDAC1 (1:1,000; cat. no. sc-390996; Santa Cruz Biotechnology, Inc.) for $12 \mathrm{~h}$ at $4^{\circ} \mathrm{C}$. Following primary antibody incubation, membranes were incubated with horseradish peroxidase-conjugated secondary antibodies for $1 \mathrm{~h}$ at room temperature, including anti-rabbit $\operatorname{IgG}(1: 5,000$; cat. no. 7074) and anti-mouse $\operatorname{IgG}(1: 5,000$; cat. no. 7076; both Cell Signaling Technology, Inc.). Protein bands were visualized using the ECL Western Blotting Substrate (EMD Millipore) and Tanon-5200 Chemiluminescence Imager (Tanon Science and Technology Co., Ltd.).

Reverse transcription-quantitative polymerase chain reaction $(R T-q P C R)$. Total RNA was extracted from HUVECs using the TRIzol ${ }^{\circledR}$ reagent (Thermo Fisher Scientific, Inc.). Reverse transcription was performed using a reverse transcription kit (Vazyme Biotech Co., Ltd.). qPCR was subsequently performed using a StepOnePlus ${ }^{\mathrm{TM}}$ Real-Time PCR System (Applied Biosystems; Thermo Fisher Scientific, Inc.) with THUNDERBIRD ${ }^{\circledR}$ SYBR $^{\circledR}$ qPCR master mix (Vazyme Biotech Co., Ltd.). The Thermocycling conditions were as follows: Initial polymerase activation step at $95^{\circ} \mathrm{C}$ for $30 \mathrm{sec}$; followed by 40 cycles at $95^{\circ} \mathrm{C}$ for $10 \mathrm{sec}$ and $60^{\circ} \mathrm{C}$ for $30 \mathrm{sec}$. A final stage was performed at $95^{\circ} \mathrm{C}$ for $15 \mathrm{sec}, 60^{\circ} \mathrm{C}$ for
$60 \mathrm{sec}$ and $95^{\circ} \mathrm{C}$ for $15 \mathrm{sec}$. The following primer sequences were used for qPCR: HK2 forward, 5'-TGCCACCAGACT AAACTAGACG-3' and reverse, 5'-CCCGTGCCCACAATG AGAC-3'; PPAR $\gamma$ forward, 5'-GGGATCAGCTCCGTGGAT CT-3' and reverse, 5'-TGCACTTTGGTACTCTTGAAG TT-3'; Bcl-2 forward, 5'-GGTGGGGTCATGTGTGTGG-3' and reverse, 5'-CGGTTCAGGTACTCAGTCATCC-3'; and GAPDH forward, 5'-GGAGCGAGATCCCTCCAAAAT-3' and reverse, 5'-GGCTGTTGTCATACTTCTCATGG-3'. The relative expression levels of target genes were quantified using the $2^{-\Delta \Delta \mathrm{Cq}}$ method (21) and normalized to the internal reference gene GAPDH.

Dual-luciferase reporter assay. HUVEC cells were seeded onto 24-well plates at a density of $1 \times 10^{5}$ cells/well and co-transfected with luciferase reporter plasmids $200 \mathrm{ng}$ pGL3-HK2, pGL3-Bcl-2 or 10 ng pRL-TK-Renilla and 100 ng pSV SPORT PPAR $\gamma$, pSV SPORT, pCF CREB, pcDNA3.1, pCMV4-p65 or pCMV4 expression vectors in $250 \mathrm{ml}$ of serum-free DMEM using Lipofectamine ${ }^{\circledR} 2000$ (Thermo Fisher Scientific, Inc.) for $4 \mathrm{~h}$ at $37^{\circ} \mathrm{C}$. Following incubation, HUVECs were cultured in DMEM supplemented with 10\% FBS for $24 \mathrm{~h}$. Following a 24-h incubation, transfected HUVECs were cultured in DMEM containing $5.5 \mathrm{mM}$ glucose for a further $48 \mathrm{~h}$. Luciferase activities were detected using the Dual-Luciferase Reporter assay system (Promega Corporation, Madison, WI, USA) and Firefly activity was normalized to Renilla luciferase activity. Three biological replicates were performed in triplicate.

Statistical analysis. Data are presented as the mean \pm standard error. Comparisons between groups were analyzed via one-way analysis of variance (GraphPad, Inc.). $\mathrm{P}<0.05$ was considered to indicate a statistically significant result. Three biological replicates were performed in triplicate.

\section{Results}

High glucose induces apoptosis in HUVECs. HUVECs exposed to high concentrations (16.5 and $33 \mathrm{mM})$ of glucose demonstrated a significantly decreased cell viability, increased cell apoptosis and cleaved caspase-3 levels, compared with HUVECs exposed to a low (5.5. $\mathrm{mM}$ ) glucose concentration (Fig. 1A-C). In addition, the mitochondrial membrane potential in HUVECs exposed to high concentrations of glucose was significantly decreased compared with HUVECs exposed to a low glucose concentration (Fig. 1D). These results suggest that exposure to high glucose may induce cell apoptosis in a mitochondria-dependent manner via impairment of mitochondrial structure and function. The change in mitochondrial permeability was confirmed by a reduced mitochondrial expression level and an unchanged cellular expression level of cytochrome c (Fig. 1E and F), which is released from the mitochondria into the cytoplasm when mitochondrial function is disrupted (22). Since mitochondrial permeability is controlled by VDAC1, which is regulated by $\mathrm{Bcl}-2$, Bax and $\mathrm{HK} 2, \mathrm{Bcl}-2$, Bax and HK2 protein expression (19) and subcellular distribution were examined. HUVECs exposed to high glucose concentrations demonstrated decreased mitochondrial and cellular protein expression levels of HK2 and Bcl-2 compared with HUVECs exposed to a low glucose concentration 
A

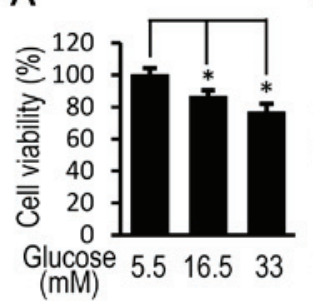

B

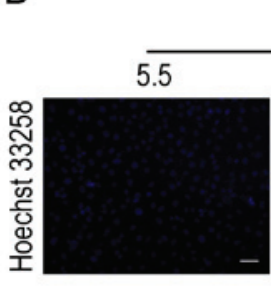

Glucose (mM)

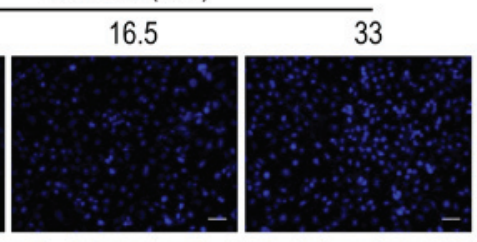

C

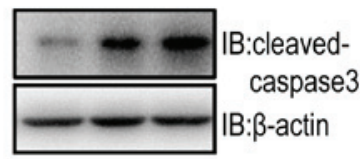

5.516 .533 Glucose(mM)

D

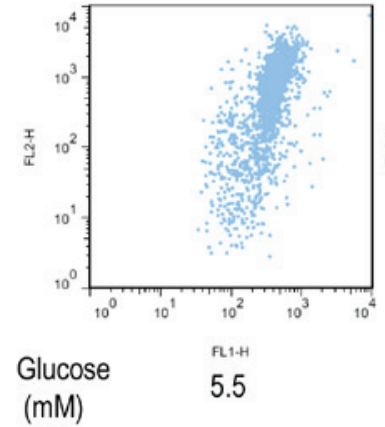

E

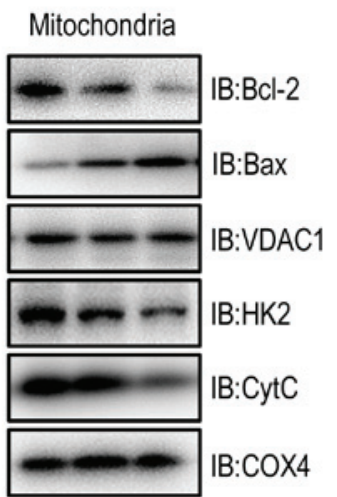

5.516 .533 Glucose(mM)

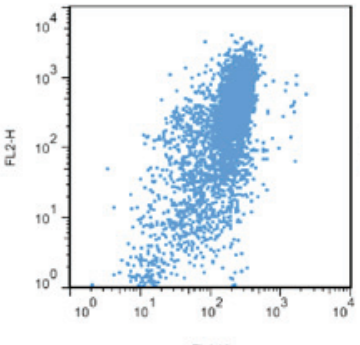

16.5

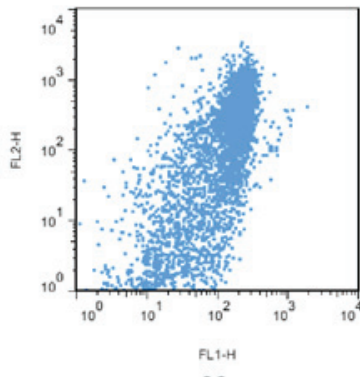

33

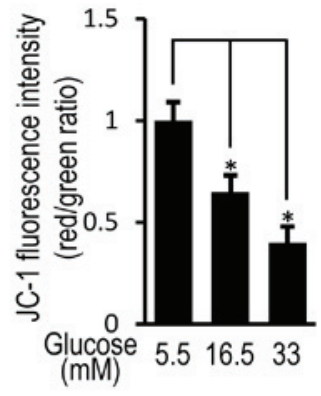

$\underset{(\mathrm{mM})}{\operatorname{Glucose}} 5.516 .533$
$\mathrm{F}$

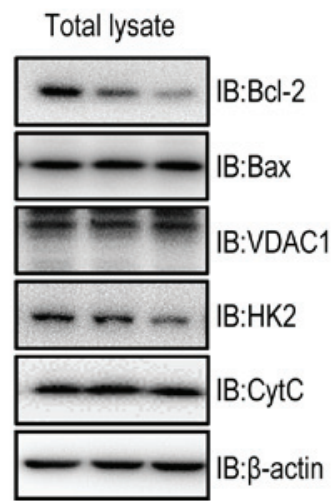

5.516 .533 Glucose(mM)
G

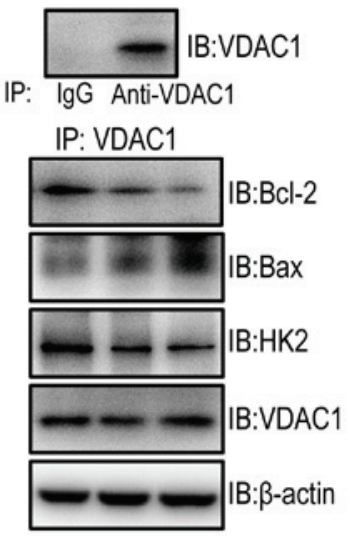

5.516 .533 Glucose(mM)

Figure 1. High glucose induces apoptosis in HUVECs by disturbing the mitochondrial membrane potential. HUVECs were cultured in DMEM containing 5.5, 16.5 or $33 \mathrm{mM}$ glucose for $72 \mathrm{~h}$. (A) MTT assay was used to examine cell viability of HUVECs. (B) Cell apoptosis was examined in HUVECs stained with the fluorescent nuclear dye Hoechst 33258 . Scale bar $=200 \mathrm{~mm}$. (C) The protein expression level of cleaved caspase-3 was determined by western blot analysis in HUVECs. (D) Mitochondrial membrane potential was examined in HUVECs following JC-1 staining and flow cytometric analysis for fluorescence emission at $530 \mathrm{~nm}$ (JC-1 FL1) and $575 \mathrm{~nm}$ (JC-1 FL2). The FL2/FL1 ratio represents mitochondrial membrane potential. (E) The protein expression level of Bcl-2, Bax, VDAC1, HK2, CytC and COX4 were determined by western blot analysis in mitochondria isolated from HUVECs. (F) The protein expression levels of Bcl-2, Bax, VDAC1, HK2 and CytC were determined by western blot analysis in HUVECs. (G) The protein expression level of Bcl-2, Bax, HK2 and VDAC1 were determined by western blot analysis in HUVECs following immunoprecipitation with VDAC1 antibody. Data are presented as the mean \pm standard error. " $\mathrm{P}<0.05$ as indicated. HUVEC, human umbilical vein endothelial cell; DMEM, Dulbecco's modified Eagle's medium; Bax, Bcl-2 associated X; VDAC1, voltage-dependent anion channel 1; HK2, hexokinase 2; CytC, cytochrome c; COX4, cytochrome c oxidase subunit 4; IB, immunoblotting.

(Fig. 1E and F). Although the mitochondrial protein level of VDAC1, as well as the cellular protein levels of VDAC1 and Bax remained unchanged, the mitochondrial protein level of Bax was increased in HUVECs exposed to high concentrations of glucose (Fig. 1E and F). Immunoprecipitation studies revealed that exposure to high glucose enhanced the interaction between VDAC1 and Bax, and reduced the interaction between VDAC1 and HK2 as well as Bcl-2 in HUVECs (Fig. 1G).

HK2 overexpression partially reverses high glucose-induced cell apoptosis. Protein expression levels of HK2 were increased in HUVECs following transfection with HK2 adenovirus compared with control adenovirus (Fig. 2A). When compared with control virus infected HUVECs, HK2 overexpression partially reversed the decrease in cell viability, enhanced cell apoptosis and activation of caspase-3, and reduced mitochondrial membrane potential of HUVECs following exposure to high levels of glucose (Fig. 2B-E). In addition, HK2 overexpression partially reversed the decreased mitochondrial and cellular protein expression levels of HK2 and Bcl-2 as well as the increased mitochondrial protein expression level of Bax observed in HUVECs compared with control virus infected HUVECs following exposure to high levels of glucose. Furthermore, HK2 overexpression suppressed cytochrome 
A

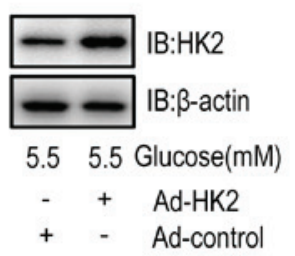

D

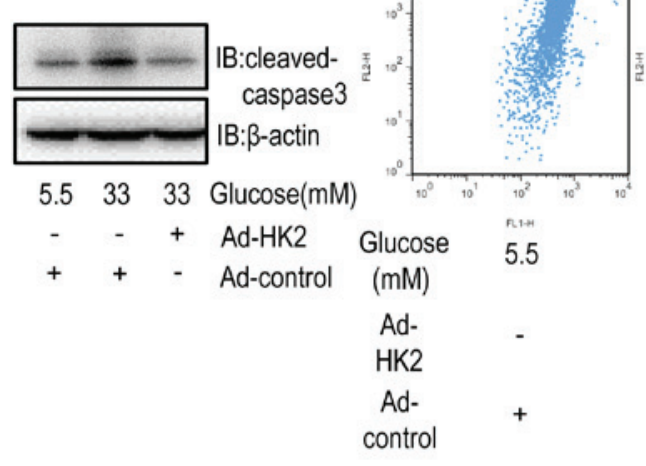

F

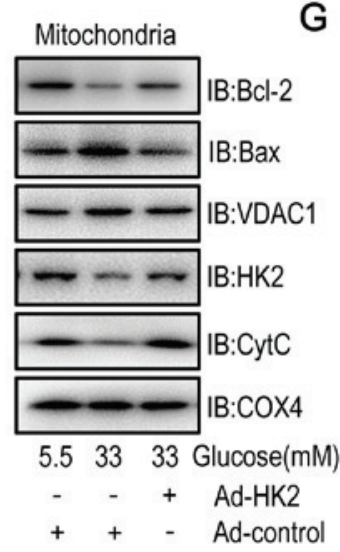

I

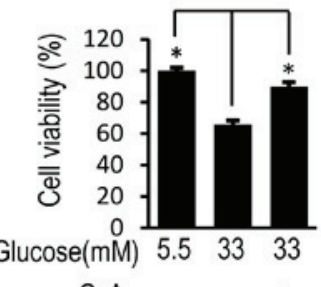

B

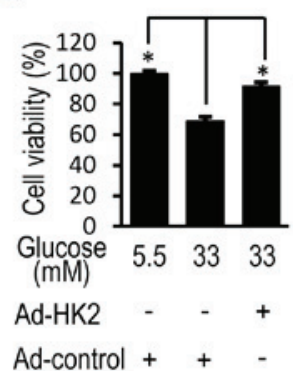

E
C

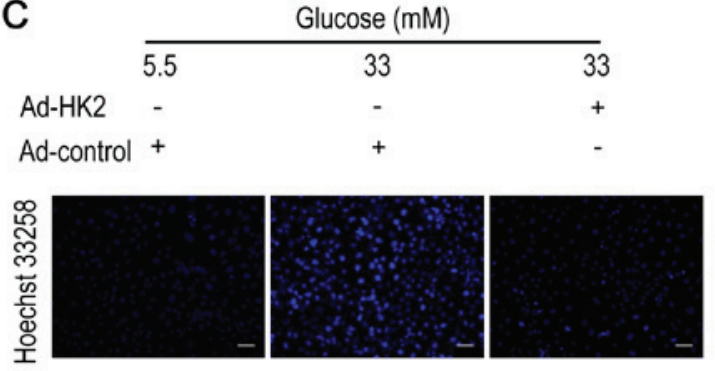

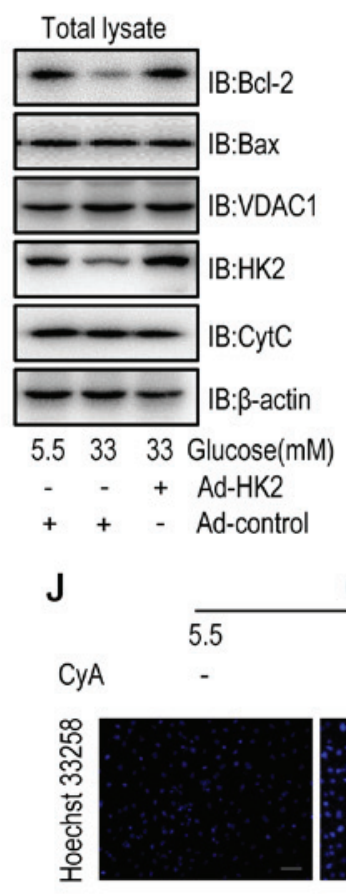

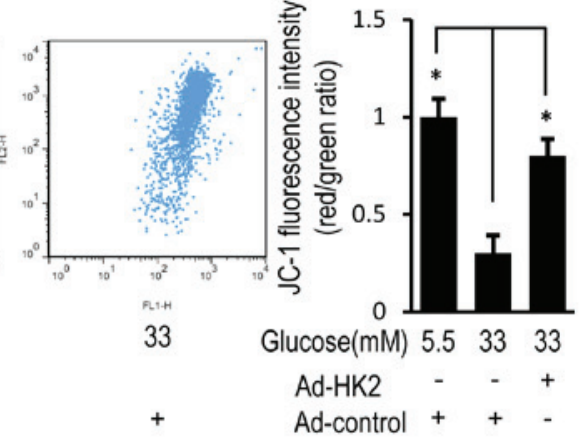

H

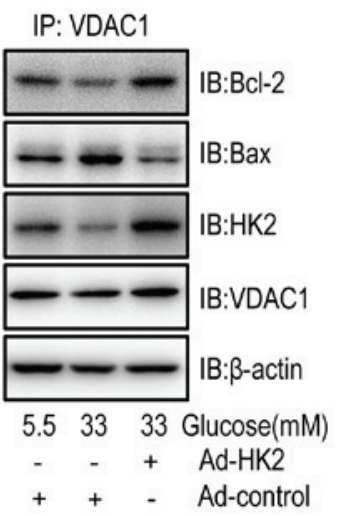

Glucose (mM)

$33 \quad 33$
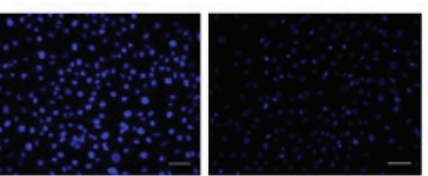

Figure 2. HK2 overexpression reverses high glucose-induced apoptosis in HUVECs. HUVECs were transfected with HK2 adenovirus or control virus for $24 \mathrm{~h}$. Following transfection, HUVECs were cultured in DMEM containing 5.5, 16.5 or $33 \mathrm{mM}$ glucose for a further $72 \mathrm{~h}$. (A) The protein expression level of HK2 was determined by western blot analysis in HUVECs. (B) MTT assay was used to examine cell viability of HUVECs. (C) Cells apoptosis was examined in HUVECs stained with the fluorescent nuclear dye Hoechst 33258. Scale bar=200 mm. (D) The protein expression level of cleaved caspase-3 was determined by western blot analysis in HUVECs. (E) Mitochondrial membrane potential was examined in HUVECs following JC-1 staining and flow cytometric analysis for fluorescence emission at $530 \mathrm{~nm}$ (JC-1 FL1) and $575 \mathrm{~nm}$ (JC-1 FL2). (F) The protein expression levels of Bcl-2, Bax, VDAC1, HK2, CytC and COX4 were determined by western blot analysis in mitochondria isolated from HUVECs. (G) The protein expression levels of Bcl-2, Bax, VDAC1, HK2 and CytC were determined by western blot analysis in HUVECs. (H) The protein expression levels of Bcl-2, Bax, HK2 and VDAC1 were determined by western blot analysis in HUVECs following immunoprecipitation with VDAC1 antibody. HUVECs were treated with $10 \mathrm{mM}$ CyA for $12 \mathrm{~h}$. Following 12-h treatment, HUVECs were cultured in DMEM containing 5.5 or $33 \mathrm{mM}$ glucose for a further $72 \mathrm{~h}$. (I) MTT assay was used to examine cell viability of HUVECs following treatment with CyA. (J) Cells apoptosis was examined in HUVECs stained with the fluorescent nuclear dye Hoechst 33258 following treatment with CyA. Scale bar $=200 \mu \mathrm{m}$. Data are presented as the mean \pm standard error. "P $<0.05$ as indicated. HK2, hexokinase 2; HUVEC, human umbilical vein endothelial cell; DMEM, Dulbecco's modified Eagle's medium; Bax, Bcl-2 associated X; VDAC1, voltage-dependent anion channel 1; CytC, cytochrome c; COX4, cytochrome c oxidase subunit 4 ; IB, immunoblotting. 
A

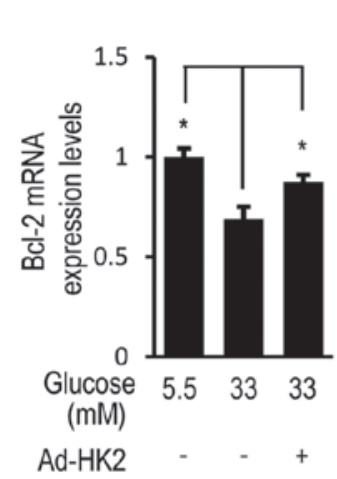

B

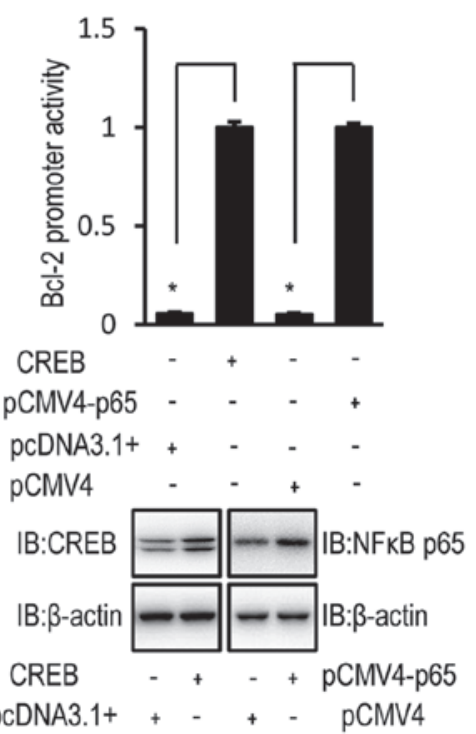

C

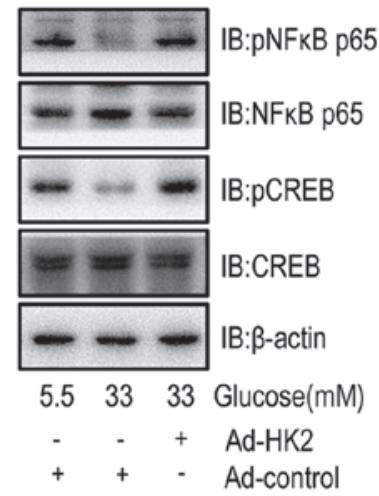

Figure 3. High glucose downregulates Bcl-2 expression via HK2 expression. HUVECs were transfected with HK2 adenovirus or control virus for 24 h. Following transfection, HUVECs were cultured in DMEM containing 5.5, 16.5 or $33 \mathrm{mM}$ glucose for $72 \mathrm{~h}$. (A) The relative mRNA expression level of Bcl-2 was determined by RT-qPCR in HUVECs. (B) HUVECs were co-transfected with pCF CREB (or pcDNA3.1) and pGL3-Bcl-2 (or pRL-TK-Renilla) or pCMV4-p65 (NF- $\mathrm{BB}$ ) (or pCMV4) and pGL3-Bcl-2 (or pRL-TK-Renilla) for $48 \mathrm{~h}$ and luciferase activity was detected using the Dual-Luciferase reporter assay. The protein expression of CREB or NF- $\mathrm{B}$ were increased in HUVECs following transfection with pCF CREB or pCMV4-p65 compared with pcDNA3.1 or

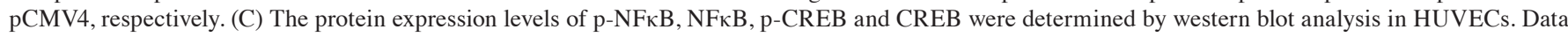
are presented as the mean \pm standard error. ${ }^{*} \mathrm{P}<0.05$ as indicated. HK2, hexokinase 2; HUVEC, human umbilical vein endothelial cell; DMEM, Dulbecco's modified Eagle's medium; NFאB, nuclear factor- $\kappa \mathrm{B}$; CREB, cyclic AMP response element binding protein; RT-qPCR, reverse transcription-quantitative polymerase chain reaction; IB, immunoblotting.

c release (Fig. 2F and G). Furthermore, immunoprecipitation assays revealed that $\mathrm{HK} 2$ overexpression enhanced the interactions between VDAC1 and HK2 as well as Bcl-2, and attenuated the interactions between VDAC1 and Bax (Fig. 2H). CyA, a known inhibitor of mitochondrial permeability transition (23), was used to examine the effect of mitochondrial permeability on cell viability and apoptosis in HUVECs exposed to a high concentration of glucose. Treatment with CyA partially reversed the decrease in cell viability and the increase in cell apoptosis in HUVECs exposed to a high glucose concentration, similarly to the aforementioned HK2 overexpression (Fig. 2I and J).

High glucose-induced Bcl-2 downregulation is reversed by HK2 upregulation. HK2 overexpression partially restored the relative mRNA expression level of Bcl-2 in HUVECs exposed to high concentrations of glucose (Fig. 3A). Luciferase assays confirmed the transcriptional regulation of $\mathrm{Bcl}-2$ by $\mathrm{CREB}$ and NF- $\kappa \mathrm{B}$ p65 in HUVECs (Fig. 3B). Furthermore, the phosphorylation level of CREB and $N F-\kappa B$, as an indicator of activation, was examined by western blot analysis in HUVECs. HK2 overexpression reversed the high glucose-induced effect on the activation status of phosphorylated CREB and $\mathrm{NF}-\kappa \mathrm{B}$ in HUVECs (Fig. 3C).

High glucose reduces HK2 transcription via downregulation of PPAR $\gamma$ in HUVECs. To investigate the mechanisms underlying the inhibitory effect of high glucose on HK2 expression, the relative mRNA and protein expression levels of HK2 were determined by RT-qPCR and western blotting, respectively, in HUVECs exposed to high and low glucose concentrations.
The relative mRNA and protein expression levels of HK2 were decreased in HUVECs exposed to high glucose concentrations compared with HUVECs exposed to a low glucose concentration (Fig. 4A and B). In addition, when MG132, a cell-permeable proteasome inhibitor, was used to block HK2 protein degradation, a high glucose-induced decrease in HK2 protein expression level was observed (Fig. 4B). These results suggest that high glucose may regulate HK2 expression at a transcriptional level. Furthermore, PPAR $\gamma$ is reported to be a transcription factor for HK2 (24). In the current study, luciferase activity confirmed the transcriptional regulation of HK2 by PPAR $\gamma$ (Fig. 4C). Although HUVECs exposed to high glucose concentrations demonstrated decreased mRNA and protein expression levels of endogenous PPAR $\gamma$ (Fig. 4D and E), high glucose had no obvious effect on the protein expression level of flag-tagged PPAR $\gamma$ level in HUVECs (Fig. 4F).

\section{Discussion}

Hyperglycemia-induced apoptosis of vascular endothelial cells serves a role in the pathogenesis of both macrovascular and microvascular complications of diabetes (25). In the present study, an in vitro model of chronic hyperglycemia was established in HUVECs following exposure to high glucose, in order to investigate the potential mechanisms and therapeutic targets for diabetes-induced vascular complications.

In the present study, high glucose-induced apoptosis in HUVECs was associated with the release of mitochondrial cytochrome $\mathrm{c}$, as a result of the increase in mitochondrial membrane permeability demonstrated by the reduced mitochondrial membrane potential. VDAC1 plays a role in regulating 
A

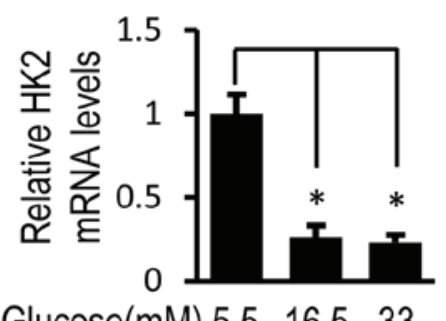

Glucose(mM) $5.5 \quad 16.5 \quad 33$
B

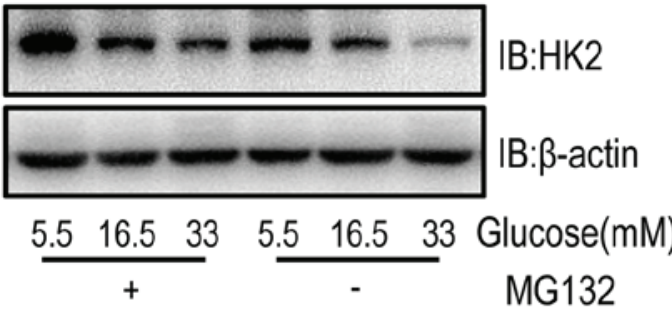

E

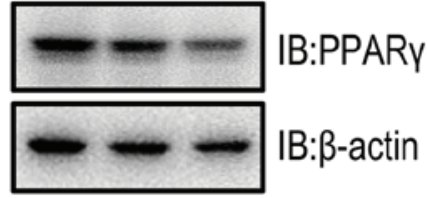

5.516 .533 Glucose(mM)
C

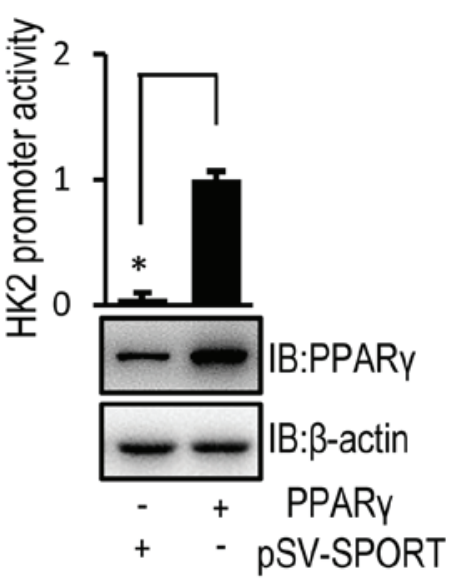

F

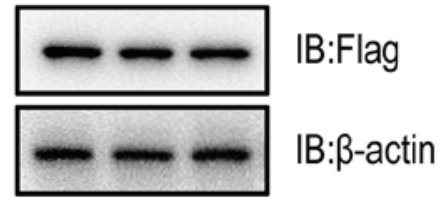

$\begin{array}{llll}5.5 & 16.533 & \text { Glucose(mM) }\end{array}$

+++ Flag-PPARY

Figure 4. High glucose inhibits HK2 transcription of HK2 via PPAR $\gamma$ downregulation in HUVECs. (A) HUVECs were cultured in DMEM containing 5.5, 16.5 or $33 \mathrm{mM}$ glucose for $72 \mathrm{~h}$ and the relative mRNA expression level of HK2 was determined by RT-qPCR. (B) HUVECs were cultured in DMEM containing $5.5,16.5$ or $33 \mathrm{mM}$ glucose for $72 \mathrm{~h}$ followed by treatment with MG132 (50 $\mu \mathrm{M})$ for $8 \mathrm{~h}$. Following 8 -h treatment, the protein expression level of HK2 was determined by western blot analysis. (C) HUVECs were co-transfected with pSV SPORT PPAR $\gamma$ (or pSV-SPORT) and pGL3-HK2 (or pRL-TK-Renilla) into cells for $48 \mathrm{~h}$ and luciferase activity was detected using the Dual-Luciferase assay. HUVECs were cultured in DMEM containing 5.5, 16.5 or 33 mM glucose for $72 \mathrm{~h}$. (D) The relative mRNA expression level of PPAR $\gamma$ was determined by RT-qPCR in HUVECs. (E) The protein expression level of PPAR $\gamma$ was determined by western blot analysis in HUVECs. (F) HUVECs were transfected with the Flag tagged PPAR $\gamma$ for $24 \mathrm{~h}$. Following transfection, HUVECs were cultured in medium containing 5.5, 16.5 or $33 \mathrm{mM}$ glucose for $72 \mathrm{~h}$. The protein expression level of Flag was determined by western blot analysis in HUVECs. Data are presented as the mean \pm standard error. ${ }^{*} \mathrm{P}<0.05$ as indicated. HK2, hexokinase 2; PPAR $\gamma$, peroxisome proliferator-activated receptor $\gamma$; HUVEC, human umbilical vein endothelial cell; DMEM, Dulbecco's modified Eagle's medium; RT-qPCR, reverse transcription-quantitative polymerase chain reaction; MG132, cell-permeable proteasome inhibitor; IB, immunoblotting.

mitochondrial membrane permeability (26), following exposure to high glucose, and its function is regulated by $\mathrm{HK} 2, \mathrm{Bcl}-2$ and Bax (27). While HK2 and Bcl-2 reduce mitochondrial permeability, Bax increases mitochondrial permeability (16-18). In the current study, high glucose-induced downregulation of mitochondrial and cellular HK2 and Bcl-2 expression, and therefore decreased interactions with VDAC1. In addition, high glucose induced upregulation of mitochondrial Bax by enhancing interactions with VDAC1 without affecting total lysate Bax expression levels. As HK2 and Bax competitively bind to VDAC1 (28), the decreased protein expression level of HK2 is likely to be affect the interactions between Bax and VDAC1. It was therefore hypothesized that downregulation of HK2 was involved in high glucose-induced cell apoptosis. To examine whether HK2 was involved in high glucose-induced cell apoptosis, HK2 was overexpressed in HUVECs exposed to high glucose. HK2 overexpression partially suppressed high glucose-induced cell apoptosis, by reducing mitochondrial Bax and its interaction with VDAC1. The antiapoptotic effect of HK2 may be achieved by directly competing with Bax for binding to VDAC1, as there was no upregulation of intracellular Bax observed. Furthermore, HK2 overexpression increased the expression, mitochondrial abundance and interaction of $\mathrm{Bcl}-2$ with $\mathrm{VDAC} 1 . \mathrm{Bcl}-2$ is an apoptotic regulator which plays an antiapoptotic role by binding to the N-terminal domain of VDAC1 (29). Therefore, upregulation of Bcl-2 may also contribute to the antiapoptotic effect of HK2.

Consistent with the findings of the current study, previous studies demonstrated that HK2 upregulated Bcl-2 expression in several types of cancer cell lines (30), through the activation of CREB, a Bcl-2 transcription factor, via phosphorylation (31). Despite the lack of studies regarding the effect of long-term exposure to high glucose on CREB phosphorylation, the current study indicated that high glucose effectively attenuated CREB phosphorylation in HUVECs. In addition, the current study demonstrated that high glucose attenuated phosphorylation of $\mathrm{NF}-\kappa \mathrm{B}$, another $\mathrm{Bcl}-2$ transcriptional factor (32). However, HK2 overexpression partially reversed the high glucose-induced inhibition of $N F-\kappa B$ and CREB phosphorylation. Therefore, the inhibition of CREB and $\mathrm{NF}-\kappa \mathrm{B}$ phosphorylation by high glucose may reduce $\mathrm{Bcl}-2$ expression, however this was partially reversed by HK2 overexpression. The underlying mechanism of CREB and NF- $\kappa \mathrm{B}$ regulation by high glucose and HK2 remains unknown.

To investigate the underlying mechanism by which high glucose reduces HK2 expression in HUVECs, the mRNA and protein levels of HK2 were examined. The current study demonstrated that high glucose reduced the transcription of 
HK2 without affecting its protein degradation rate. As high glucose may increase the proteasome-mediated degradation of HK2, MG132 was used to prevent this protein degradation, which resulted in a reduced difference of protein levels between high and low glucose treatment. A previous study revealed that PPAR $\gamma$ is an important transcription factor for HK2 (25), which was confirmed in the current study using a luciferase assay. As was the case with HK2, high glucose reduced PPAR $\gamma$ expression by decreasing its mRNA level. Therefore, high glucose-induced downregulation of PPAR $\gamma$ led to suppressed HK2 transcription. However, the mechanism underlying high glucose-mediated regulation of PPAR $\gamma$ expression in HUVECs requires further investigation.

In conclusion, high glucose reduced HK2 expression by suppressing the expression of the HK2 transcription factor (PPAR $\gamma$ ), which subsequently reduced $\mathrm{Bcl}-2$ expression in HUVECs. These changes weakened the interaction of mitochondrial VDAC1 with $\mathrm{HK} 2$ and Bcl-2 leading to the enhanced binding of Bax to VDAC1, which increased mitochondrial membrane permeability and induced cell apoptosis. It is hypothesized that upregulating HK2 or PPAR $\gamma$ may reduce vascular endothelial cell apoptosis and prevent diabetes-induced vascular complications.

\section{Acknowledgements}

The authors would like to thank Dongping Wei (The First Affiliated Hospital of Nanjing, Nanjing, China) for the luciferase reporter plasmids pGL3-HK2 and pGL3-Bcl-2, and the pCF CREB, pCMV4 and pSV-SPORT expression vectors. The authors would also like to acknowledge Dr Bruce Spiegelman (Dana-Farber Cancer Institute, Harvard Medical School) and Dr Warner Greene (Howard Hughes Medical Institute, Department of Medicine, Duke University Medical Center) pSV SPORT PPAR $\gamma$ and pCMV4 p65 expression vectors, respectively.

\section{Funding}

The present study was funded by grants from the Natural Science Foundation of Jiangsu Province of China (grant no. BK20151015) and the Jiangsu Health International Exchange Program.

\section{Availability of data and materials}

The datasets used and/or analyzed during the present study are available from the corresponding author on reasonable request.

\section{Authors' contributions}

JZ performed experiments and prepared the manuscript. YG performed the cell apoptosis analysis. WG and XZ performed the cell viability assay. MP designed and directed the experiments, and wrote the manuscript. All authors read and approved the final manuscript.

\section{Ethics approval and consent to participate}

Not applicable.

\section{Patient consent for publication}

Not applicable.

\section{Competing interests}

The authors declare that they have no competing interests.

\section{References}

1. Wang N, Cheng J, Han B, Li Q, Chen Y, Xia F, Jiang B, Jensen MD and Lu Y: Exposure to severe famine in the prenatal or postnatal period and the development of diabetes in adulthood: An observational study. Diabetologia 60: 262-269, 2017.

2. Ervasti J, Virtanen M, Lallukka T, Pentti J, Kjeldgård L, Mittendorfer-Rutz E, Tinghög $\mathrm{P}$ and Alexanderson K: Contribution of comorbid conditions to the association between diabetes and disability pensions: A population-based nationwide cohort study. Scand J Work Environ Health 42: 209-216, 2016.

3. Guo R, Liu B, Wang K, Zhou S, Li W and Xu Y: Resveratrol ameliorates diabetic vascular inflammation and macrophage infiltration in $\mathrm{db} / \mathrm{db}$ mice by inhibiting the NF- $\mathrm{NB}$ pathway. Diab Vasc Dis Res 11: 92-102, 2014.

4. Wang Q, Zhang M, Torres G, Wu S, Ouyang C, Xie Z and Zou MH: Metformin suppresses diabetes-accelerated atherosclerosis via the inhibition of Drp1-mediated mitochondrial fission. Diabetes 66: 193-205, 2017.

5. Sturm A, Mollard V, Cozijnsen A, Goodman CD and McFadden GI: Mitochondrial ATP synthase is dispensable in blood-stage Plasmodium berghei rodent malaria but essential in the mosquito phase. Proc Natl Acad Sci U S A 112: 10216-10223, 2015.

6. Gottlieb RA: Cell death pathways in acute ischemia/reperfusion injury. J Cardiovasc Pharmacol Ther 16: 233-238, 2011.

7. Balk J, Leaver CJ and Mccabe PF: Translocation of cytochrome c from the mitochondria to the cytosol occurs during heat-induced programmed cell death in cucumber plants. FEBS Lett 463: 151-154, 1999.

8. Wen Y, Li W, Poteet EC, Xie L, Tan C, Yan LJ, Ju X, Liu R, Qian H, Marvin MA, et al: Alternative mitochondrial electron transfer as a novel strategy for neuroprotection. J Biol Chem 286: 16504-16515, 2011.

9. Shalaeva DN, Dibrova DV, Galperin MY and Mulkidjanian AY: Modeling of interaction between cytochrome $\mathrm{c}$ and the WD domains of Apaf-1: Bifurcated salt bridges underlying apoptosome assembly. Biol Direct 10: 29, 2015.

10. Safi SZ, Batumalaie K, Mansor M, Chinna K, Mohan S, Karimian H, Qvist R, Ashraf MA and Yan GO: Glutamine treatment attenuates hyperglycemia-induced mitochondrial stress and apoptosis in umbilical vein endothelial cells. Clinics (Sao Paulo) 70: 569-576, 2015.

11. Shoshan-Barmatz V, Krelin Y and Shteinfer-Kuzmine A: VDAC1 functions in $\mathrm{Ca}(2+)$ homeostasis and cell life and death in health and disease. Cell Calcium 69: 81-100, 2017.

12. Ben-Hail D, Begas-Shvartz R, Shalev M, Shteinfer-Kuzmine A, Gruzman A, Reina S, De Pinto V and Shoshan-Barmatz V: Novel compounds targeting the mitochondrial protein VDAC1 inhibit apoptosis and protect against mitochondria dysfunction. J Biol Chem 291: 24986-25003, 2016

13. Shoshan-Barmatz V and Mizrachi D: VDAC1 (voltage-dependent anion channel 1). Atlas Genet Cytogenet Oncol Haematol, 2012.

14. Shoshanbarmatz V and Mizrachi D: VDAC1: From structure to cancer therapy. Front Oncol 2: 164, 2012.

15. Shi Y, Chen J, Weng C, Chen R, Zheng Y, Chen Q and Tang H: Identification of the protein-protein contact site and interaction mode of human VDAC1 with Bcl-2 family proteins. Biochem Biophys Res Commun 305: 989-996, 2003.

16. Zhou H, Zhang Y, Hu S, Shi C, Zhu P, Ma Q, Jin Q, Cao F, Tian F and Chen Y: Melatonin protects cardiac microvasculature against ischemia/reperfusion injury via suppression of mitochondrial fission-VDAC1-HK2-mPTP-mitophagy axis. J Pineal Res 63: e12413, 2017.

17. Sharpe JC, Arnoult D and Youle RJ: Control of mitochondrial permeability by Bcl-2 family members. Biochim Biophys Acta 1644: 107-113, 2004. 
18. Pastorino JG, Chen ST, Tafani M, Snyder JW and Farber JL: The overexpression of Bax produces cell death upon induction of the mitochondrial permeability transition. J Biol Chem 273: 7770-7775, 1998

19. Abu-Hamad S, Arbel N, Calo D, Arzoine L, Israelson A, Keinan N Ben-Romano R, Friedman O and Shoshan-Barmatz V: The VDAC1 $\mathrm{N}$-terminus is essential both for apoptosis and the protective effect of anti-apoptotic proteins. J Cell Sci 122: 1906-1916, 2009.

20. Zitman-Gal T, Green J, Pasmanik-Chor M, Golan E, Bernheim J and Benchetrit S: Vitamin D manipulates miR-181c, miR-20b and miR-15a in human umbilical vein endothelial cells exposed to a diabetic-like environment. Cardiovasc Diabetol 13: 8, 2014.

21. Livak KJ and Schmittgen TD: Analysis of relative gene expression data using real-time quantitative PCR and the 2(-Delta Delta) C(T)) method. Methods 25: 402-408, 2001.

22. Gogvadze V, Orrenius S and Zhivotovsky B: Multiple pathways of cytochrome c release from mitochondria in apoptosis. Biochim Biophys Acta 1757: 639-647, 2006.

23. Varela AT, Gomes AP, Simões AM, Teodoro JS, Duarte FV, Rolo AP and Palmeira CM: Indirubin-3'-oxime impairs mitochondrial oxidative phosphorylation and prevents mitochondrial permeability transition induction. Toxicol Appl Pharmacol 233: 179-185, 2008.

24. Panasyuk G, Espeillac C, Chauvin C, Pradelli LA, Horie Y, Suzuki A, Annicotte JS, Fajas L, Foretz M, Verdeguer F, et al: PPAR $\gamma$ contributes to PKM2 and HK2 expression in fatty liver. Nat Commun 3: 672, 2012.

25. Amore A, Cirina P, Conti G, Cerutti F, Bagheri N, Emancipator SN and Coppo R: Amadori-configurated albumin induces nitric oxide-dependent apoptosis of endothelial cells: A possible mechanism of diabetic vasculopathy. Nephrol Dial Transplant 19: 53-60, 2004

26. Zamarin D, García-Sastre A, Xiao X, Wang R and Palese P: Influenza virus $\mathrm{PB} 1-\mathrm{F} 2$ protein induces cell death through mitochondrial ANT3 and VDAC1. Plos Pathog 1: e4, 2005.
27. Huang L, Han J, Ben-Hail D, He L, Li B, Chen Z, Wang Y, Yang Y, Liu L, Zhu Y, et al: A new fungal diterpene induces VDAC1-dependent apoptosis in bax/bak-deficient cells. J Biol Chem 290: 23563-23578, 2015.

28. Tomasello MF, Guarino F, Reina S, Messina A and De Pinto V: The voltage-dependent anion selective channel 1 (VDAC1) topography in the mitochondrial outer membrane as detected in intact cell. Plos One 8: e81522, 2013.

29. Monaco G, Decrock E, Arbel N, van Vliet AR, La Rovere RM, De Smedt H,Parys JB, Agostinis P, Leybaert L, Shoshan-Barmatz V and Bultynck G: The BH4 domain of anti-apoptotic Bcl-XL, but not that of the related $\mathrm{Bcl}-2$, limits the voltage-dependent anion channel 1 (VDAC1)-mediated transfer of pro-apoptotic $\mathrm{Ca} 2+$ signals to mitochondria. J Biol Chem 290: 9150-9161, 2015.

30. Rho M, Kim J, Jee CD, Lee YM, Lee HE, Kim MA, Lee HS and Kim WH: Expression of type 2 hexokinase and mitochondria-related genes in gastric carcinoma tissues and cell lines. Anticancer Res 27: 251-258, 2007.

31. Meller R, Minami M, Cameron JA, Impey S, Chen D, Lan JQ, Henshall DC and Simon RP: CREB-mediated Bcl-2 protein expression after ischemic preconditioning. J Cereb Blood Flow Metab 25: 234-346, 2005.

32. Sheu ML, Ho FM, Yang RS, Chao KF, Lin WW, Lin-Shiau SY and Liu SH: High glucose induces human endothelial cell apoptosis through a phosphoinositide 3-kinase-regulated cyclooxygenase-2 pathway. Arterioscler Thromb Vasc Biol 25: 539-545, 2005.

c) (7) $\Theta$ This work is licensed under a Creative Commons Attribution-NonCommercial-NoDerivatives 4.0 International (CC BY-NC-ND 4.0) License. 